\title{
Análise do comportamento da função pulmonar e força muscular respiratória em coronariopatas idosos submetidos à revascularização do miocárdio
}

\author{
Elayne Kelen de Oliveira' \\ Vinicius Zacarias Maldaner da Silva² \\ Aída Luiza Ribeiro Turquetto ${ }^{3}$ \\ Maurício Milani ${ }^{4}$
}

\section{Resumo}

A cirurgia de revascularização do miocárdio (RM) pode ocasionar redução dos volumes e capacidades pulmonares e alterações músculo-esqueléticas, que podem aumentar a permanência coronariopata idoso no hospital, expondo-o a uma maior possibilidade de infecções e complicações clínicas. Esse estudo tem por objetivo avaliar as mudanças na função pulmonar e na força muscular respiratória do período pré-operatório ao $6^{\circ}$ dia de pós-operatório em coronariopatas submetidos à RM acima de 60 anos. Foi realizado um estudo de caráter coorte prospectivo no Instituto do Coração (InCor)-DF, do qual foram avaliados 9 pacientes (idade 67,20 $\pm 6,34$ anos). Houve uma redução significativa dos valores de CVF ( $\mathrm{p}=0.0040$ ), VEF1 ( $\mathrm{p}=0.0012$ ), enquanto as demais variáveis não apresentam diferença significativa no período analisado. Esses dados sugerem uma redução dos valores de função pulmonar que permanecem baixos até o $6^{\circ}$ dia de pós-operatório, além de caracterizar uma alteração restritiva nesse grupo de pacientes.

Palavras-chave: Função respiratória. Respiração. Cirurgia cardíaca.

\footnotetext{
${ }^{1}$ Bolsista - PIC/UniCEUB. Graduanda do curso de Fisioterapia no UniCEUB.

${ }^{2}$ Orientador PIC/UniCEUB. Docente do curso de Fisioterapia no UniCEUB.

${ }^{3}$ Supervisora da Fisioterapia do Instituto do Coração, InCor-DF.

${ }^{4}$ Médico Cardiologista, doutorando da Universidade de São Paulo.
} 


\section{Introdução}

A Doença Arterial Coronariana (DAC) é uma das principais causas mundiais de morte, sendo a primeira na população de 60 anos ou mais. São em média $3.8 \mathrm{mi}-$ lhões de homens e 3.4 milhões de mulheres que morrem todo ano pela doença. A incidência está aumentando em países em desenvolvimento, em parte pelo aumento da longevidade, urbanização e mudanças de hábitos diários (WHO, 2004).

O tratamento clínico para as doenças coronarianas pode ser dividido em medicamentoso e cirúrgico, sendo a revascularização do miocárdio um entre os procedimentos mais realizados (PIEGAS et al.,2004). Esta cirurgia está associada a uma prevalência significativa de complicações renais, músculo-esqueléticas, neurológicas, e pulmonares, ocasionando deficiência na função respiratória. Esses fatores estão relacionados diretamente ao desenvolvimento de morbidades nos pacientes, no período pós-operatório (STILLER et al., 1997; SILVA, 2004).

Essas alterações músculo-esqueléticas e neurológicas estão relacionadas às cargas mecânicas aplicadas ao paciente durante a cirurgia, como retração esternal, canulação venosa central, desvascularização do esterno e posicionamento do paciente (ELANSARY, 1995). Os danos na parede torácica devido a esternotomia, associados ao processo de envelhecimento, geram queda na força muscular respiratória, o que pode afetar o desempenho ventilatório e comprometer a capacidade funcional dos idosos (RODRIGUES et al., 2006).

Além disso, a anestesia geral está associada à depressão do estímulo respiratório, resultando em hipoventilação alveolar e redução da capacidade residual funcional (BORGUI; SILVA, 2004).

Alterações nesses parâmetros funcionais pulmonares estão relacionadas a um aumento no tempo de internação hospitalar, o que gera uma menor rotatividade de pacientes e aumenta o custo dos centros hospitalares (EL-ANSARY, 2000).

Em vista dessa situação, tem-se como objetivo do trabalho avaliar a função pulmonar e a força muscular respiratória em coronariopatas pacientes submetidos a esternotomia mediana. 


\section{Metodologia}

Foi realizado um estudo de caráter coorte prospectivo, no qual foi avaliada a função pulmonar e a força muscular respiratória em coronariopatias submetidas a revascularização do miocárdio, isolada por meio de esternotomia mediana em ambos os gêneros (masculino e feminino), com idade acima de 60 anos, atendidos no Instituto do Coração, InCor-DF, no período de março a agosto de 2007.

Foram incluídos 16 pacientes, sendo a amostra composta por 6 mulheres e 10 homens, com idade média de 68,4 $\pm 6,17$ anos, que foram avaliados no período préoperatório. Os pacientes foram informados sobre os procedimentos a serem realizados e assinaram um termo de consentimento livre e esclarecido para participar deste estudo, em atendimento à Resolução 196/96, do Conselho Nacional de Saúde. Este estudo foi aprovado pelo Comitê de Ética e Pesquisa do Centro Universitário de Brasília - UniCEUB e pelo Comitê de Ética e Pesquisa do InCor-DF. Foram excluídos da pesquisa os pacientes que apresentaram idade inferior a 60 anos, instabilidade clínica, indicação de cirurgia de revascularização do miocárdio associada a outro procedimento, fração de ejeção $<25 \%$, seqüelas neurológicas associadas e/ou que não assinaram o termo de consentimento livre e esclarecido.

Os pacientes incluídos no estudo foram submetidos a um questionário clínico o qual foi complementado com os dados do prontuário do mesmo, que constituiu uma coleta sobre a sua saúde em geral, presença de fatores de risco e elucidação dos procedimentos e exames realizados antes da cirurgia.

Após os questionários, foram mensuradas a altura $(\mathrm{m})$ e massa $(\mathrm{Kg})$ do paciente. Em seguida, foi realizada a espirometria, conforme já realizado por Sant’anna Junior et al (2006), Moraes et al (2006) e Nasrala et al (2006). Foi utilizado o espirômetro portátil da marca Micro Medical, modelo Microl, ab 3500 (Rochester, England). Foi realizada a manobra de Capacidade Vital Forçada, com o paciente sentado, com as costas apoiadas no encosto posterior da cadeira, flexão de quadril e joelho em $90^{\circ} \mathrm{e}$ com os pés totalmente em contato com o chão, sendo todas as coletas realizadas no mesmo modelo de cadeira e seguindo os procedimentos técnicos, critérios de aceitabilidade e reprodutibilidade, como recomendado pela Sociedade Torácica Americana (ATS, 2002). A técnica foi realizada até serem obtidos os valores de pelo menos três manobras, sem fuga aérea, detectada pelo aparelho e, o mesmo, escolheu a curva com 
o melhor desempenho. As variáveis do exame que serão utilizadas no estudo são: Capacidade Vital Forçada (CVF), Volume Expiratório Forçado, no $1^{\circ}$ Segundo $\left(\mathrm{VEF}_{1)} \mathrm{e}\right.$ índice de Tiffenau $\left(\mathrm{VEF}_{1} / \mathrm{CVF}\right)$. Foram medidos os valores absolutos, expressos em condições Body Temperature and Pressure Saturation (BTPS, $100 \%$ de UR e $37^{\circ} \mathrm{C}$ ), e calculados os percentuais relativos aos preditos para sexo, altura e idade, seguindo tabela descrita por Pereira et al (1991), já gravadas na memória do aparelho.

Após a espirometria, foi mensurada a força muscular respiratória por meio de um manovacuômetro, como já realizado por Cramer et al (2006), Sant’anna Junior et al (2006), Moraes et al (2006), Nasrala et al (2006) e Simões et al (2006). Foi utilizado o manovacuômetro da marca $\mathrm{CRTL}^{\circledR}$, escalonado -120 a $120 \mathrm{cmH}_{2} \mathrm{O}$, de acordo com metodologia proposta por Black \& Hyatt (1969). O teste foi realizado com o paciente sentado do mesmo modo como descrito para o teste espirométrico. O maior valor sustentado obtido foi então escolhido, desde que não fosse o da última medida (BELLINETTI; THOMSON, 2006). Todos os testes de espirometria e manovacuometria foram realizados por um único avaliador e realizados no período pré-operatório e no $6^{\circ}$ dia de pós- operatório.

Para a análise estatística, foi utilizado o teste não paramétrico de Wilcoxon para a comparação dos valores de espirometria e força muscular respiratória. Foram considerados como diferença significativa os valores de $\mathrm{p}<0,05$.

\section{Resultados}

Durante o período de março a agosto de 2007, foram internados 46 pacientes para a realização de cirurgia de revascularização do miocárdio no InCor-DF. Para o nosso estudo, foram excluídos 28 pacientes, sendo destes, 18 pacientes com idade inferior a 60 anos (44-59 anos, 52,22 $\pm 4,10$ anos), 5 pacientes que não realizariam a cirurgia de revascularização do miocárdio isolada (colocação e troca de prótese valvar associada ou marcapasso cardíaco), 4 pacientes que estavam com instabilidade clínica no período pré-operatório (um paciente submetido à revascularização de urgência e três que estavam com instabilidade hemodinâmica), um paciente com a fração de ejeção $<25 \%$, um paciente com seqüelas de um acidente vascular encefálico (AVE) e um paciente que não assinou o termo de consentimento. 
Foram coletados os dados de 16 pacientes no período pré-operatório, sendo a amostra composta por 6 mulheres e 10 homens, com idade média de 68,4 $\pm 6,17$ anos, 7 (43,75\%) ex-tabagistas, 7 (43,75\%) com diabetes mellitus, 10 (62,50\%), com infarto agudo do miocárdio (IAM) prévio, 11 (68,75\%) com hipertensão arterial sistêmica, $11(68,75 \%)$ pacientes com dislipidemia, com o período de internação préoperatória de $8 \pm 5$ dias.

Dos 16 pacientes incluídos, 7 pacientes foram excluídos no transcorrer do estudo (entre o período pré-operatório e o sexto dia pós-operatório), devido a uma desistência, dois óbitos, uma seqüela de AVE perioperatório e uma instabilidade clínica no $6^{\circ}$ dia pós-operatório. Sendo a amostra final constituída de 09 pacientes. A Tabela 1 mostra as características gerais da amostra inicial e final, que foi constituída por 5 pacientes do sexo masculino (55,56\%), 5 que apresentavam diabetes $(55,56 \%)$, 3 ex-tabagistas (33,33\%), 7 que apresentavam dislipidemia (77,78\%) e o tempo médio de internação foi de $14,6 \pm 5,28$ dias.

Tabela 1 - Características demográficas e clínicas

\begin{tabular}{lccc}
\hline \multicolumn{2}{c}{ Variáveis } & Pré-operatório & $6^{\circ} \mathrm{PO}$ \\
\hline Número total & & 16 & 9 \\
\hline Idade (anos) ${ }^{*}$ & & $68,40 \pm 6,17$ & $67,20 \pm 6,34$ \\
\hline \multirow{2}{*}{ Sexo } & Masculino & $10(62,50 \%)$ & $5(55,56 \%)$ \\
\cline { 2 - 4 } & Feminino & $6(37,50 \%)$ & $4(44,44 \%)$ \\
\hline Ex-tabagistas & $7(43,75 \%)$ & $3(33,33 \%)$ \\
\hline Diabetes mellitus & $7(43,75 \%)$ & $5(55,56 \%)$ \\
\hline IAM prévio & $10(62,50 \%)$ & $5(55,56 \%)$ \\
\hline HAS & $11(68,75 \%)$ & $7(77,78 \%)$ \\
\hline Dislipidemia & $11(68,75 \%)$ & $7(77,78 \%)$ \\
\hline Sedentarismo & $6(37,50 \%)$ & $5(55,56 \%)$ \\
\hline
\end{tabular}

*Valores expressos em Média \pm Desvio Padrão. PO = Pós-Operatório; IAM = Infarto Agudo do Miocárdio; HAS = Hipertensão Arterial Sistêmica.

Os resultados dos valores espirométricos coletados no pré-operatório e no sexto dia do pós-operatório estão descritos na Tabela 2. Após a análise estatística, 
constata-se uma queda significativa da VEF1 ( $\mathrm{p}=0,0012)$ e da CVF $(\mathrm{p}=0,0040)$, quando comparada os valores do período pré-operatório com o sexto dia. Já as variáveis Tiffenau e PFE não obtiveram correlação significativa entre os dois períodos.

Tabela 2 - Valores espirométricos

\begin{tabular}{cccc}
\hline $\begin{array}{c}\text { Variáveis } \\
\text { (\%Prev) }\end{array}$ & Pré-operatório $^{*}$ & $\mathbf{6}^{\circ} \mathbf{P O}{ }^{*}$ & $\mathbf{P}$ \\
\hline VEF1 & $87,89 \pm 16,64$ & $58,22 \pm 13,00$ & $0,0012^{\circ}$ \\
\hline CVF & $83,78 \pm 20,38$ & $55,22 \pm 9,12$ & $0,0040^{\circ}$ \\
\hline Tiffenau & $104,78 \pm 10,59$ & $102,78 \pm 11,28$ & 0,6271 \\
\hline PFE & $70,22 \pm 21,18$ & $55,00 \pm 20,42$ & 0,1135 \\
\hline
\end{tabular}

*Valores expressos em Média \pm Desvio Padrão. ${ }^{\circ}$ Valores significativos, $p<0,005$. VEF1 $=$ Volume Expiratório Forçado no $1^{\circ}$ segundo; $\mathrm{CVF}=$ Capacidade Vital Forçada; PFE = Pico de Fluxo Expiratório.

O gráfico 1 ilustra o comportamento da CVF do período pré-operatório, ao $6^{\circ}$ dia de pós-operatório, do qual obteve uma diferença significativa para essa variável.

\section{Discussão}

Neste estudo, foram encontradas reduções significativas nas variáveis CVF e VEF1, indicando um padrão restritivo, assim como estudos de Silva (2004), Barbosa \& Carmona (2004) e Pinto et al (1998).

Essa diminuição dos valores de CVF e VEF1 sugere alterações na função pulmonar, estas podem estar associadas a diversos fatores, como o tipo de incisão cirúrgica, técnica de anestesia empregada, dor pós-operatória e posicionamento do dreno pleural (EL-ANSARY, 2000; GUIZILINI et al., 2004). 


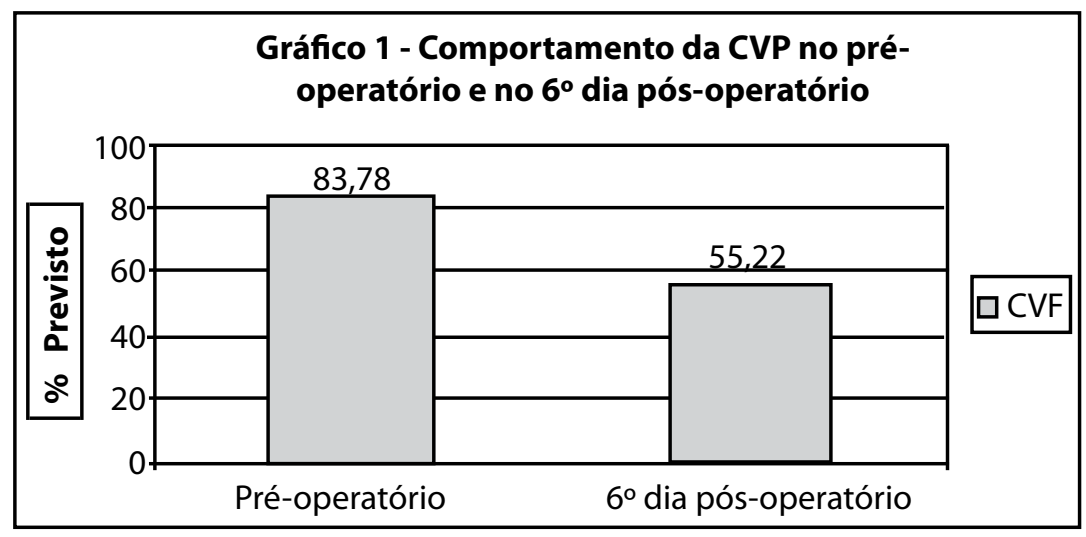

Essas alterações têm relação com procedimentos transoperatórias e pósoperatórias. Taggart et al (1993) relatam que a esternotomia mediana altera a complacência da caixa torácica, gerando alterações na Curva Pressão X Volume do sistema respiratório, aumentando a retração elástica, dificultando a geração de volumes no sistema. Esse procedimento também dificulta a expansibilidade torácica, o que pode ocasionar respirações mais superficiais, com redução do volume corrente.

Frowfelter (1983) descreve alterações no tônus diafragmático, após cirurgias de revascularização do miocárdio, relacionados ao tipo de anestesia empregado e a utilização da Circulação Extra Corpórea (CEC), que pode reduzir o fluxo sanguíneo a essa região. O Tônus diafragmático é de suma importância para a manutenção da Capacidade Residual Funcional (CRF), pois ele minimiza o efeito da pressão intra-abdominal sobre a área pulmonar, o que impede a compressão pulmonar e formação de atelectasias. Nesse grupo de pacientes, a redução do Tônus diafragmático está em aproximadamente $20 \%$, o que pode reduzir os volumes pulmonares.

Outro fator descrito por Frowfelter (1983) é o fechamento de pequenas vias aéreas em virtude do efeito anestésico. Esse fechamento diminuiria a ventilação alveolar que seria outro fator para predispor o aparecimento de atelectasias.

Nozawa et al (2003) descrevem a dor pós-operatória como um fator importante na redução dos volumes pulmonares. A dor estaria associada à menor 
expansibilidade torácica e respirações superficiais, com redução no volume corrente. No nosso trabalho, tivemos a preocupação de analisar o dor dos pacientes pesquisados, no $6^{\circ}$ dia de pós-operatório, por meio de uma escala analógica de dor. Em nossa amostra, não houve nenhum paciente que apresentasse níveis significativos de dor (acima de 6), demonstrando que esse fator não está relacionado à manutenção da redução dos volumes pulmonares nesse período.

Barbosa \& Carmona (2004) descrevem as alterações pleurais como importante fator para a redução dos volumes pulmonares. Por manipulação da caixa torácica e mediastino, a incidência de lesões pleurais em revascularização do miocárdio é elevada.

Guizilini et al (2004) descrevem que a posição do dreno também influencia nos volumes pulmonares. Os drenos posicionados, na região sub-xifóidea, apresentam maior redução do que os drenos mediastinais laterais. Todos os pacientes incluídos no estudo apresentaram dreno sub-xifóideo, fator que pode predispor a redução dos volumes pulmonares.

Em relação à força muscular inspiratória e expiratória, este trabalho encontrou resultados diferentes dos trabalhos de Silva (2004), Cramer (2006), Rodrigues et al (2006), Sant’anna Junior et al (2006), Simões et al., (2006) e Nasrala et al (2006). Dois fatores podem ter influenciado nesse achado. O primeiro é a amostra reduzida deste estudo ( $n=9)$, que pode influenciar nos achados estatísticos. O segundo fator pode ser a metodologia empregada na mensuração da força muscular pela manovacuometria utilizada nesses estudos. Todos os outros estudos realizaram 3 medidas para identificar a PImax e PEmax, destacando sempre o maior valor obtido. Porém o estudo de Fiore Junior et al (2005) sugere que o efeito aprendizagem deve ser considerado quando é realizada a avaliação de pressões respiratórias máximas. Com esse fator, há sugestão de que o valor máximo de PImáx é obtido após a realização de cinco manobras. Na avaliação da PEmáx são necessárias, no mínimo onze manobras, para que o valor máximo seja atingido. Provavelmente, com 3 medidas, obtém-se valores menores dessas variáveis, o que influenciaria nesses valores. Outro dado é em que tempo do período pósoperatório foram mensurados esses valores. A maioria dos trabalhos utilizou o período de coleta entre o $3^{\circ}$ ao $4^{\circ}$ dia de pós-operatório, período no qual pode ser influenciada pelos efeitos da anestesia, da dor pós-operatória e o tempo de fisioterapia recebido. 
Esse estudo apresenta algumas limitações, tais como o tempo para o desenvolvimento do estudo, problemas internos ocorridos na instituição onde foi realizada a coleta, além do tempo de treinamento para o avaliador identificar essas variáveis, acabaram influenciando no número amostral e conseqüentemente nos resultados do estudo.

\section{Conclusão}

Os pacientes idosos submetidos à cirurgia de revascularização do miocárdio apresentaram alterações espirométricas significativas no período pós-operatório, na capacidade vital forçada e no volume expiratório forçado no $1^{\circ}$ segundo. Novos estudos devem ser realizados para identificar o comportamento dessas variáveis em um maior intervalo de tempo e identificação dos principais fatores relacionados a essa alteração. Também se sugere que devem ser desenvolvidas estratégias para a rápida recuperação desses valores, como também intervenções pré-operatórias para que a queda desses valores no pós-operatório seja amenizada.

\section{Pulmonary function and respiratory muscle strengh behavior analysis in aging coronariopaties submitted to coronary artery bypass grafting}

\section{Abstract}

The surgery of coronary artery bypass grafting can take to several functional alterations, like: pulmonary function, with pulmonary capacities decreased, musculoskeletal complications, which could increase permanence of the aging at the Hospital, displaying to high possibility of infections and clinical complications. The aim of this study was evaluated the functional parameters, quality of life and depression in patients with Coronary Artery Disease submitted to surgery of myocardial grafting in InCor-DF in 4 times: preoperative, 6, 30, 60 days after surgery. 9 patients with age $67,20 \pm 6,34$ years finished the study. There was significant reduction in the spirometric variables FVC $(\mathrm{p}=0.0040)$, FEV1 $(\mathrm{p}=0.0012)$, while the other variables didn't have significant difference between evaluated time. These data suggest the decreasing in the values of pulmonary function, which remains low until the $6^{\text {th }}$ post-operatory day, beyond to characterize this group of patients in restrictive alteration.

Keywords: Respiratory function. Respiration. Heart surgery. 


\section{Referências}

AMERICAN THORACIC SOCIETY (ATS). ATS Statement: Guidelines for the SixMinute Walk Test. American Journal of Respiratory and Critical Care Medicine, [S.l.], v. 166, p. 111-117, 2002.

BARBOSA, R.; CARMONA, M. Avaliação da função pulmonar de pacientes submetidos a cirurgia de revascularização do miocárdio com circulação extracorpórea. Revista Brasileira de Anestesiologia, Rio de Janeiro, v. 52, p. 689-699, 2002.

BELLINETTI, L. M.; THOMSON, J. C. Respiratory muscle evaluation in elective thoracotomies and laparotomies of the upper abdomen. Jornal Brasileiro de Pneumologia, São Paulo, v. 32, n. 2, p. 99-105, 2006.

BLACK, L. F.; HYATT, R. E. Maximal respiratory pressures: normal values and relationship to age and sex. The American Review of Respiratory Disease, United States, v. 99, n. 5, p. 696-702, 1969.

CRAMER, A. S. Avaliação da força muscular respiratória e amplitude torácica no pré e pós-operatório de revascularização do miocárdio. Revista Brasileira de Fisioterapia, São Carlos, supl., set. 2006. Anais XIII Simpósio Internacional de Fisioterapia Respiratória.

EL-ANSARY, D. Musculoskeletal and neurological complications following coronary artery bypass grafting: a comparison between saphenous vein and internal mammary artery grafting. Australian Journal of Physiotherapy, Austrália, n. 46, p. $19-25,2000$.

FIORE JUNIOR, J. et al. Quantas medidas são necessárias para que sejam obtidos valores máximos de pressões respiratórias máximas. In: Revista Brasileira de Fisioterapia, Ouro Preto, supl., p. 115, 2004. XII Simpósio Internacional de Fisioterapia Respiratória.

FROWFELTER, D. Fisioterapia Cardiopulmonar: princípios e prática. 1. ed. São Paulo: Atheneu, 1983.

GUIZILINI, S. et al. Efeitos do local de inserção do dreno pleural na função pulmonar no pós-operatório de cirurgia de revascularização do miocárdio. Revista Brasileira de Cirurgia Cardiovascular, São José do Rio Preto, v. 19, n. 1, p. 47-54, 2004.

MORAES, C. et al. Estudo do comportamento da função pulmonar e da força muscular respiratória em pacientes submetidos a cirurgia cardíaca. Revista Brasileira 
de Fisioterapia, São Carlos, supl., set. 2006. Anais XIII Simpósio Internacional de Fisioterapia Respiratória.

NASRALA, M. L. S. et al. Avaliação da $\mathrm{PI}_{\mathrm{MÁX}}, \mathrm{PE}_{\mathrm{MÁX}}$ e capacidade vital em pacientes antes e após cirurgia cardíaca com esternotomia mediana. Revista Brasileira de Fisioterapia, São Carlos, supl., set. 2006. Anais XIII Simpósio Internacional de Fisioterapia Respiratória.

NOZAWA, E. Identificação dos fatores de risco que influenciam no desmame de ventilação mecânica em pacientes traqueostomizados após cirurgia cardíaca. Arquivos Brasileiros de Cardiologia, São Paulo, v. 80, n. 3, p. 301-305, 2003.

PEREIRA, C. A. C. et al. Valores de referência para espirometria em uma amostra da população brasileira. Jornal Brasileiro de Pneumologia, Brasília, v. 18, n. 1, p. 10-22, 1992.

PIEGAS et al. II Diretriz sobre o tratamento do infarto agudo do miocárdio. Arquivos Brasileiros de Cardiologia, São Paulo, v. 81, supl. 1, jan. 2004.

PINTO, A. M. R. et al. Estudo da função pulmonar em pacientes submetidos a revascularização do miocárdio sem circulação extracorpórea com derivação intraluminal. Revista do Colégio Brasileiro de Cirurgiões, Rio de Janeiro, v. 25, n. 6, p. 363-368, 1998.

RODRIGUES, A. M. S. et al. Análise das correlações entre a capacidade funcional, força inspiratória e ventilometria no pós-operatório de cirurgia cardíaca. Revista Brasileira de Fisioterapia, São Carlos, supl., set. 2006. Anais XIII Simpósio Internacional de Fisioterapia Respiratória.

SANT'ANNA JUNIOR, M. et al. Avaliação longitudinal no pré e pós- operatório de cirurgia de revascularização miocárdica: impacto sobre a função pulmonar. Revista Brasileira de Fisioterapia, São Carlos, supl., set. 2006. Anais XIII Simpósio Internacional de Fisioterapia Respiratória.

SILVA, A. Borghi et al. Comportamento da função pulmonar e da força muscular respiratória em pacientes submetidos à revascularização do miocárdio. Revista Brasileira de Terapia Intensiva, São Paulo, v. 16, p. 155-159, 2004.

SIMÕES, C. et al. Estudo do comportamento da função pulmonar e da força muscular respiratória em pacientes submetidos a cirurgia cardíaca. Revista Brasileira de Fisioterapia., São Carlos, supl., set. 2006. Anais XIII Simpósio Internacional de Fisioterapia Respiratória. 
TAGGART, D. et al. Respiratory dysfunction after uncomplicated cardiopulmonary bypass. The Annals of Thoracic Surgery, n. 556, p. 1123-1128, 1993.

WORLD HEALTH ORGANIZATION (WHO). Global burden of coronary heart disease. In: MACKAY, J.; MENSAH, G. Atlas of Heart Disease and Stroke. 2004. 